\title{
LA PROTECTION DES SAVOIRS TRADITIONNELS PAR LES DROITS DE PROPRIÉTÉ INTELLECTUELLE
}

Henrique Mercer $^{1}$

\section{RÉSUMÉ}

Cet article vise à montrer la nature des savoirs traditionnels, autant que leur importance. Ces savoirs méritant d'être protégés juridiquement, on propose que la protection soit faite par le biais des droits de propriété intellectuelle. En vue de démontrer la pertinence de cette proposition, on mène une analyse des alternatives possibles de protection dans le cadre des droits de propriété intellectuelle.

\section{ABSTRACT}

This article means to display the nature of traditional knowledge, as well as it importance. Traditional knowledge deserves to be legally protected, and it proposes that the protection take place trough intellectual property rights. To demonstrate the pertinence of this proposition, it does an analysis of the possible alternatives of protection within the intellectual property rights.

MOTS-CLÉS : Savoirs traditionnels, propriété intellectuelle, protection

KEYWORDS: Traditional knowledge, intellectual property, protection

\section{INTRODUCTION}

Après la signature de la Convention sur la diversité biologique (CDB) de l'Organisation des Nations Unies $(\mathrm{ONU})^{2}$, la reconnaissance des savoirs

\footnotetext{
${ }^{1}$ Avocat, étudiant à la maitrise en Droit international de l'Université du Québec à Montréal, membre du NDI et du NUPESUL (UFPR), spécialiste en Droit International (PUC-PR) et en Diplomatie et négociations internationales (Unibrasil).
}

Revista Brasileira de Direito Internacional, Curitiba, v.7, n.7, jan./jun.2008 
traditionnels des communautés autochtones et locales ${ }^{3}$ est devenue une question centrale dans la suite des négociations de la Convention parce que ces savoirs sont investis d'un rôle fondamental dans la protection de la biodiversité et dans l'instauration d'un marché de ressources génétiques. Pour cette raison, ils ont été qualifiés par Pinton et Grenand ${ }^{4}$ comme « patrimoine culturel à respecter, information à protéger et marchandise intangible à valoriser » dans la nouvelle économie de la connaissance. Ces deux dernières qualifications touchent intimement aux droits de propriété intellectuelle.

La Convention ne fait aucune prescription d'un régime à être adopté par les parties afin de protéger et de valoriser les savoirs traditionnels. Toutefois, c'est une tâche urgente. Historiquement, les savoirs traditionnels sont en train d'être appropriés, adaptés et exploités par des chercheurs et par l'industrie, principalement ceux du Nord. Même si la plus grande partie des savoirs traditionnels se trouvent dans les pays du Sud, c'est le Nord qui s'en enrichit ${ }^{5}$.

Le présent travail a comme objectif de montrer les régimes capables d'effectuer cette protection et cette valorisation.

\subsection{LA NATURE DES SAVOIRS TRADITIONNELS}

Les autochtones sont les peuples qui vivent dans un même environnement depuis des générations. Ces populations vivent dans une profonde relation avec leur environnement, si bien qu'elles préservent constamment la nature et la biodiversité dans leur territoire. Elles pratiquent, en quelque sorte, un développement durable ${ }^{6}$ même avant de la création de cette notion. Depuis beaucoup les autochtones ont développé plusieurs

\footnotetext{
2 ORGANISATION DES NATIONS UNIES. Convention sur la diversité biologique. Rio de Janeiro, 5 junho 1992. [Online] Disponível em: <www.cbd.int $>$. Acesso em: jan. 2008.

${ }_{3}$ Pour le but de ce travail, les communautés autochtones et locales seront désignées par communautés autochtones.

4 PINTON, Florence \& GRENAND, Pierre. Savoirs traditionnels, populations locales et ressources globalisées. In : AUBERTIN, Catherine et al (dir.). Les marches de la biodiversité. Paris: IRD Éditions, 2007, p. 165.

${ }^{5}$ MÜLLER, Andréia N.. A proteçāo dos conhecimentos tradicionais por meio das indicações geográficas. In: RODRIGUES JR, Edson B.. \& POLIDO, Fabrício (dir.). Propriedade intelectual: novos paradigmas internacionais, conflitos e desafios. Rio de Janeiro: Elsevier, 2007, p. 302.

${ }^{6}$ Dont le concept sera vu à la suite.
}

Revista Brasileira de Direito Internacional, Curitiba, v.7, n.7, jan./jun.2008 
connaissances et pratiques fondées dans l'emploi des ressources naturelles de leur environnement, qui les ont aidés à se nourrir, à s'habiller et à se soigner.

Les savoirs traditionnels comprennent les informations sur les usages des éléments biologiques pour des fins médicinales et agricoles, les processus de productions, les dessins, la littérature, la musique, les rituels et d'autres techniques et arts $^{7}$. La plupart des savoirs traditionnels ne sont pas contemporains, mais ils ne sont pas statiques non plus. Ils sont en évolution constante, et toujours en train de produire de nouvelles informations comme conséquence de l'adaptation au changement des circonstances. Les savoirs traditionnels peuvent appartenir aux individus (dans le cas de pratiques de cure et de rituels, par exemple), à quelques membres d'une communauté ou à tous les membres - savoir commun. Certains savoirs traditionnels peuvent être utilisés hors du contexte de leurs communautés d'origine, mais d'autres non, car ils peuvent avoir des composants spirituels particuliers à la communauté qui les maîtrisent ${ }^{8}$.

Les savoirs traditionnels sont des créations collectives qui dérivent de la pensée et de la vie pratique de la communauté et ils sont transmis de génération en génération. Ils constituent la composante intangible/immatérielle de la biodiversité. Selon Moretti et Aubertin ${ }^{9}$, les communautés autochtones sont détentrices de savoirs traditionnels relatifs aux ressources génétiques susceptibles d'orienter les processus d'innovation. D'après Shiva ${ }^{10}$, selon certaines estimations, la valeur courante dans le marché mondial pour les plantes médicinales identifiées grâce aux pistes données par les communautés autochtones serait de l'ordre de 43 milliards de dollars. À part la valeur

7 ORGANISATION MONDIALE DE LA PROPRIÉTÉ INTELLECTUELLE. World Intellectual Property Organization (WIPO) Draft Report on Fact-finding Missions on Intellectual Property and Traditional Knowledge (1998-1999) - Draft for Comment - July 3, 2000. [Online] Disponível em: <www.wipo.int>. Acesso em : nov. 2007.

${ }^{8}$ CORREA, Carlos Maria. Traditional knowledge and intellectual property: issues and options surrounding the protection of traditional knowledge - A discussion paper. Genebra: Quaker United Nations Office, 2001. [Online] Disponível em: www.iucn.org/themes/pbia/themes/trade/training/TK\%20and\%20Intellectual\%20Property.pdf. Acesso em nov. 2007, p. 4.

${ }^{9}$ MORETTI, Christian \& AUBERTIN, Catherine. Stratégies des firmes pharmaceutiques : la bioprospection en question. In: AUBERTIN, Catherine et al (dir.). Les marches de la biodiversité. Paris: IRD Éditions, 2007, p. 27.

10 SHIVA, Vandana. Bioporataria: a pilhagem da natureza e do conhecimento. Petrópolis: Vozes, 2001.

Revista Brasileira de Direito Internacional, Curitiba, v.7, n.7, jan./jun.2008 
économique apportée aux industries pharmaceutiques et cosmétiques, le fait que ces produits soient développés avec des plantes par de processus découlant de savoirs traditionnels ajoute aussi de la valeur commerciale à l'image de ces industries ${ }^{11}$.

\subsection{L'IMPORTANCE DE LA PROTECTION DES SAVOIRS} TRADITIONNELS

En raison de l'évolution de la biotechnologie ${ }^{12}$, les savoirs traditionnels ont actuellement un grand potentiel économique; ils contribuent à développer des produits dans le domaine de la pharmacologie, du cosmétique, de l'industrie alimentaire, agricole ${ }^{13}$, énergétique (dans la production de combustible d'origine végétale) ${ }^{14}$, chimique et textile. Tous ces domaines sont très importants dans le commerce international.

Barbosa $^{15}$ indique trois aspects importants des savoirs traditionnels:

11 MORETTI, Christian \& AUBERTIN, Catherine. Stratégies des firmes pharmaceutiques : la bioprospection en question. In: AUBERTIN, Catherine et al (dir.). Les marches de la biodiversité. Paris: IRD Éditions, 2007, p. 48.

${ }_{12}$ Le terme biotechnologie est utilisé pour définir toutes les technologies qui utilisent des systèmes biologiques ou des organismes vivants et leurs dérivés pour créer ou modifier produits ou processus. Selon l'article 2 de la CDB biotechnologie est «toute application technologique qui utilise des systèmes biologiques, des organismes vivants, ou des dérivés de ceux-ci, pour réaliser ou modifier des produits ou des procédées à usage spécifique » (ORGANISATION DES NATIONS UNIES. Convention sur la diversité biologique. Rio de Janeiro, 5 junho 1992. [Online] Disponível em: <www.cbd.int>. Acesso em: jan. 2008.). Santé Canada définit biotechnologie comme « un terme qui englobe une large variété d'activités scientifiques dans plusieurs sphères, telles que les aliments, la santé et l'agriculture. Elle inclut l'utilisation d'organismes vivants, ou de parties d'organismes vivants, pour engendrer de nouvelles méthodes de production et la fabrication de nouveaux produits » (SANTE CANADA. Biotechnologie. [Online] Disponível em: < http://www.hc-sc.gc.ca/sr-sr/biotech/index-fra.php>. Acesso em: jun. 2008.

${ }^{13}$ Le savoir traditionnel des agriculteurs autochtones joue un rôle central dans le développement des nouvelles variétés végétales en échelle globale (CORREA, Carlos Maria. Traditional knowledge and intellectual property: issues and options surrounding the protection of traditional knowledge - A discussion paper. Genebra: Quaker United Nations Office, 2001. [Online] Disponível em: www.iucn.org/themes/pbia/themes/trade/training/TK\%20and\%20Intellectual\%20Property.pdf. Acesso em nov. 2007, p. 3.

${ }^{14}$ DEL NERO, Patrícia Aurélia. A propriedade Intelectual da Biotecnologia. In: CARVALHO, Patrícia Luciane (dir.). Propriedade intelectual: estudos em homenagem à professora Maristela Basso. Curitiba : Juruá, 2005, p.353.

${ }^{15}$ BARBOSA, Denis B..Uma introduçāo à propriedade intelectual. Rio de Janeiro: Lumen Juris, 2003, p. 784.

Revista Brasileira de Direito Internacional, Curitiba, v.7, n.7, jan./jun.2008 
a) La découverte de nouvelles espèces

b) L'indication des composants chimiques ou biologiques pertinents;

c) Techniques de préservation et gestion de l'environnement.

Une autre raison importante pour la protection des savoirs traditionnels c'est le fait que,

Après quelques dénonciations de dépôts de brevets prétendument issus de l'observation sur terrain de pratiques séculaires, les connaissances relatives aux utilisations traditionnelles des plantes sont jugées extrêmement précieuses et menacées [par les pays du Sud riches en biodiversité $^{16}$.

Les connaissances traditionnelles ne sont pas un trésor à maintenir caché. Au contraire, il y aurait tout l'intérêt à les faire partager et l'initiative privée est bienvenue pour contribuer à cette diffusion. La question à résoudre c'est de procurer une façon d'exploiter ces savoirs sans exploiter les autochtones. Comment garantir que les autochtones reçoivent le prix juste pour leur savoir et qu'ils ne soient pas volés par les industries et par les laboratoires?

Une solution possible - qui fera l'objet de la présente réflexion - c'est la protection des savoirs traditionnels par les droits de propriété intellectuelle. Selon Correa $^{17}$, les raisons pour la protection de savoirs traditionnels peuvent être résumées aux points suivants :

- Égalité : Les savoirs traditionnels ont une valeur économique, mais, étant en dehors du système de protection des droits de propriété intellectuelle, leurs créateurs ne reçoivent pas la rémunération à laquelle ils ont droit pour l'appropriation de leur connaissance par une autre partie. Donc, la protection serait nécessaire pour apporter

\footnotetext{
${ }^{16}$ AUBERTIN, Catherine et al (dir.). Les marches de la biodiversité. Paris: IRD Éditions, 2007, p. 15.

${ }^{17}$ CORREA, Carlos Maria. Traditional knowledge and intellectual property: issues and options surrounding the protection of traditional knowledge - A discussion paper. Genebra: Quaker United Nations Office, 2001. [Online] Disponível em: www.iucn.org/themes/pbia/themes/trade/training/TK\%20and\%20Intellectual\%20Property.pdf. Acesso em nov. 2007, p. 5.
}

Revista Brasileira de Direito Internacional, Curitiba, v.7, n.7, jan./jun.2008 
l'égalité à des rapports qui à ce moment sont essentiellement injustes et inégaux;

- Conservation: La protection aidera dans la conservation de la diversité biologique, puisque ces savoirs sont associés au développement durable de leur environnement;

- Préservation de la pratique et des valeurs traditionnelles: La protection du savoir traditionnel peut être un cadre pour encourager le maintien des valeurs et savoirs traditionnels;

- Préservation de l'appropriation des composants du savoir traditionnel par des parties non autorisées (respect des articles : 8(j); 15; 16; et 19 de la CDB) - bref, empêcher le biopiratage;

- Promotion de l'usage et de son importance pour le développement : Assurer la compensation des détenteurs du savoir de sorte qu'ils soient stimulés à donner un usage plus large au savoir. En vue de la compensation, ils seront encouragés à conserver le savoir et à assurer l'accès futur.

\subsection{LA DIFFICULTÉ D'ACCORDER UNE PROTECTION JURIDIQUE} AUX SAVOIRS TRADITIONNELS

Si l'on considère les biens dérivés des savoirs traditionnels, comme certains médicaments, il faut bien admettre que ces savoirs ne sont pas encore protégés par le système international de propriété intellectuelle, comme sera démontré à la suite. Cela ne veut pas dire que les biens et les innovations produits avec le savoir traditionnel n'existent pas dans le commerce international ou qu'ils n'aient pas d'importance économique, politique ou juridique qui justifie la réglementation de cette propriété. Les savoirs traditionnels ne sont pas brevetables, stricto sensu, parce qu'ils ne sont pas une nouveauté, qui est une des trois conditions pour l'octroi du brevet, qui sont l'utilité, la non-évidence et la nouveauté ${ }^{18}$. Par conséquent, les savoirs

${ }^{18}$ CORREA, Carlos Maria (dir.). Temas de derecho industrial y de la competencia: propiedad intelectual y políticas de desarrollo. Buenos Aires: Ciudad Argentina, 2005, pp. 58-59.

Revista Brasileira de Direito Internacional, Curitiba, v.7, n.7, jan./jun.2008 
traditionnels ne sont pas protégés pour les mécanismes de protection existante dans les traités en vigueur (soit dans le cadre de l'OMC, OMPI, ou de l'ONU) tel comme une découverte industrielle l'est. Mais cela ne signifie pas qu'ils ne puissent pas être protégés juridiquement.

II y a, encore, une autre difficulté à surmonter : les deux sous-systèmes de protections de la propriété intellectuelle en vigueur - droits d'auteurs et propriété industrielle - utilisent une méthodologie fondée sur des valeurs individualistes de protection intellectuelle. II n'y a donc pas, dans le système international, de protection juridique pour un savoir produit dans un cadre collectiviste (les valeurs autochtones sont collectivistes, tandis que dans le capitalisme la valeur est individualiste) donc il faudra un mécanisme de protection qui prévoit une propriété collective du savoir traditionnel qui appartienne à toute une collectivité/communauté.

Dans ce qui touche aux savoirs traditionnels et leur relation avec le système de protection de la propriété intellectuelle, c'est possible d'observer que la privatisation du savoir, concept fondamental de la propriété intellectuelle, est contraire au système de valeurs et à la façon de production et reproduction du savoir des communautés autochtones, puisque dans leur culture le partage du savoir est une valeur très importante; par contre, pour eux, le savoir n'a aucune valeur économique. C'est donc difficile de trouver une façon de les rémunérer pour leur savoir traditionnel.

En tout cas, de différentes stratégies peuvent être prises pour protéger les savoirs traditionnels par les droits de propriété intellectuelle : une application des modèles de protection existants, la création d'un système sui generis ou encore la combinaison des deux ${ }^{19}$.

La protection peut aussi avoir de raisons non économiques, comme la reconnaissance morale de l'auteur. Les auteurs ont des droits économiques et moraux dans les systèmes qui suivent celui de l'Europe continentale, quoique l'Accord sur les aspects des droits de propriété intellectuelle qui touchent au

${ }^{19}$ CORREA, Carlos Maria. Traditional knowledge and intellectual property: issues and options surrounding the protection of traditional knowledge - A discussion paper. Genebra: Quaker United Nations Office, 2001. [Online] Disponível em: www.iucn.org/themes/pbia/themes/trade/training/TK\%20and\%20Intellectual\%20Property.pdf. Acesso em nov. 2007, p. 2.

Revista Brasileira de Direito Internacional, Curitiba, v.7, n.7, jan./jun.2008 
commerce $(\mathrm{ADPIC})^{20}$ n'oblige pas ses membres à suivre l'article 6 bis $^{21}$ de la Convention de Berne ${ }^{22}$ qui protège les droits moraux.

Donc, il faut protéger les droits de propriété intellectuelle des savoirs traditionnels d'une façon qui en même temps respecte les valeurs des peuples qui les ont développés. À cause du grand nombre de communautés autochtones, avec différentes valeurs, il est extrêmement difficile d'identifier les préoccupations que peut avoir chaque peuple détenteur des savoirs traditionnels. Parmi les préoccupations, l'une des plus communes est le monopole ${ }^{23}$ par celui que détient le droit de propriété intellectuelle sur le savoir, car la majorité de ces communautés ont l'ouverture et le partage du savoir comme une valeur.

\subsection{LA RELATION ENTRE LES SAVOIRS TRADITIONNELS ET LE} CONCEPT DE DÉVELOPPEMENT DURABLE

Cette discussion a lieu dans un scénario régi par le concept du développement durable. Ce principe est présent dans les Déclarations internationales sur l'environnement plus récentes ( $\mathrm{Rio}^{24}$ et Johannesburg ${ }^{25}$ ) autant que dans l'Accord instituent l'Organisation mondiale du commerce $(\mathrm{OMC})^{26}$. Selon le Principe 3 de la Déclaration de Rio: «le droit au

${ }^{20}$ ORGANISATION MONDIALE DU COMMERCE. Accord sur les aspects des droits de propriété intellectuelle qui touchent au commerce. Marrakech, 12 abril 1994. [Online] Disponível em: www.wto.org. Acesso em : jan. 2008.

${ }^{21}$ Article 6bis

Droits moraux:

1. Droit de revendiquer la paternité de l'œuvre; droit de s'opposer à certaines modifications de l'œuvre et à d'autres atteintes à celle-ci;

2. Après la mort de l'auteur;

3. Moyens de recours.

${ }_{22}$ Organisation mondiale de la propriété intellectuelle, Convention de Berne pour la protection des œuvres littéraires et artistiques.

${ }^{23}$ Droit d'exclusivité qui caractérise les droits de propriété intellectuelle conforme comme ils existent actuellement, comme sera démontré à la suite.

${ }_{24}$ Principes 1 et 4 (ORGANISATION DES NATIONS UNIES. Déclaration de Rio sur l'environnement et le développement).

${ }^{25}$ Paragraphe 5 (ORGANISATION DES NATIONS UNIES. Déclaration de Johannesburg sur le développement durable).

${ }^{26}$ Préambule (Organisation mondiale du commerce. Accord instituant l'Organisation mondiale du commerce. Marrakech, 12 abril 1994. [Online] Disponível em: www.wto.org. Acesso em: jan. 2008).

Revista Brasileira de Direito Internacional, Curitiba, v.7, n.7, jan./jun.2008 
développement doit être réalisé de façon à satisfaire équitablement les besoins relatifs au développement et à l'environnement des générations présentes et futures. $\gg 27$

La Cour internationale de justice a reconnu le développement durable comme principe de droit international dans l'arrêt Gabcikovo-Nagymaros ${ }^{28}$.

La CDB est la première convention à soutenir le développement durable ${ }^{29}$. Selon Aubertin et al, " elle protège au même temps [simultanément] l'usage durable des ressources, leur conservation, et l'équité, au moyen du partage des avantages à travers des mécanismes de marché » 30 .

II y a plusieurs définitions du développement durable. Selon le Rapport Brundtland, le développement durable est le développement qui peut « répondre aux besoins actuels sans compromettre l'aptitude des générations futures à répondre aux leurs » ${ }^{31}$.

D’après Arbour et Lavallée, « un développement durable commence avec un mode de croissance économique qui respecte les limites écologiques de la planète et qui ne met pas en danger les systèmes naturels qui nous font vivre ${ }^{32 .}$

Le monde actuel vise le développement durable, c'est-à-dire, un développement économique qui respecte la protection de l'environnement, et vice-versa. En effet, le concept de développement durable unit les concepts de développement économique et de protection environnementale d'une façon indissociable. La protection de savoirs traditionnels est un exemple évident de cette situation: l'accès aux savoirs traditionnels est important pour le

27 ORGANISATION DES NATIONS UNIES. Déclaration de Rio sur l'environnement et le développement. Rio de Janeiro, 13 junho 1992. UN Doc A/CONF.151/5/Rev. [Online] Disponível em: <www.un.org >. Acesso em: jan. 2008.

${ }^{28}$ VARELLA, Marcelo D.. Direito internacional econômico ambiental. Belo Horizonte: Del Rey, 2004, p. 36.

${ }_{29}$ AUBERTIN, Catherine \& MORETTI, Christian. La biopiraterie entre illégalité et illégitimité. In : AUBERTIN, Catherine et al (dir.). Les marches de la biodiversité. Paris: IRD Éditions, 2007, p. 115.

30 AUBERTIN, Catherine et al.. L'accès aux ressources génétiques et le partage des avantages : une question conflictuelle - exemples du Brésil et de la Bolivie. In : AUBERTIN, Catherine et al (dir.). Les marches de la biodiversité. Paris : IRD Éditions, 2007, p. 123.

${ }^{31}$ LA COMMISSION MONDIALE SUR L'ENVIRONNEMENT ET LE DÉVELOPPEMENT. Notre avenir à tous. Montréal : Éditions du Fleuve, 1988, p. 51.

32 ARBOUR, Jean-Maurice \& LAVALLÉE, Sophie.Droit international de l'environnement, Cowansville : Éditions Yvons Blais/Bruylant, 2006, p. 66.

Revista Brasileira de Direito Internacional, Curitiba, v.7, n.7, jan./jun.2008 
développement durable ${ }^{33}$ parce ces derniers sont un outil de préservation de la biodiversité (environnement) et aussi des sources d'éléments qui peuvent protéger et faciliter la vie humaine - elle aussi partie de l'environnement. En plus, ces savoirs ont le potentiel d'être un moyen pour le développement économique, soit des communautés autochtones, soit des pays où ils se trouvent ou encore des pays ou des entreprises qui les exploite commercialement.

La façon plus évidente pour protéger ces savoirs vient du domaine économique : les droits de propriété intellectuelle ${ }^{34}$.

\section{LES DROITS DE PROPRIÉTÉ INTELLECTUELLE}

Les droits de propriété intellectuelle sont parmi ceux que la société accorde aux personnes ou organisations principalement sur des œuvres créatives. Ces droits accordent à l'inventeur, pendant une durée limitée de temps, le droit d'empêcher l'utilisation de son invention sans son autorisation.

L'importance des droits de propriété intellectuelle a grandi avec l'évolution du capitalisme. Le développement de la protection aux droits de propriété intellectuelle a eu lieu presque simultanément sur les plans national et international.

Abbott ${ }^{35}$ explique:

\footnotetext{
${ }^{33}$ MÜLLER, Andréia N.. A proteçāo dos conhecimentos tradicionais por meio das indicações geográficas. In: RODRIGUES JR, Edson B.. \& POLIDO, Fabrício (dir.). Propriedade intelectual: novos paradigmas internacionais, conflitos e desafios. Rio de Janeiro: Elsevier, 2007, p. 302.

${ }^{34}$ Les droits de propriété intellectuelle sont octroyés aux personnes physiques ou morales sur les œuvres créatives (comme est le cas des savoirs traditionnels), qui sont les biens intangibles, mais susceptibles d'appréciation commerciale (raison pour laquelle la protection de ces biens intangibles est prise en charge par l'OMC).

Quand la CDB a été le premier instrument international à préconiser la protection des savoirs traditionnels, même sans faire aucune prescription sur quel régime le faire. Cette disposition a été faite accompagné par celles que visent protéger les ressources génétiques de la biodiversité par le biais de droit de propriété intellectuelle, donc ce sous-entendu que c'est celui le chemin a prendre dans la protection des savoirs traditionnels, celle est l'opinion de Pinton et Grenand (PINTON, Florence \& GRENAND, Pierre. Savoirs traditionnels, populations locales et ressources globalisées. In : AUBERTIN, Catherine et al (dir.). Les marches de la biodiversité. Paris: IRD Éditions, 2007, pp. 166-167.

${ }^{35}$ ABBOTT, Frederick M.. Intellectual property provisions of bilateral and regional trade agreements in light of U.S. federal law. In: UNCTAD-ICTSD Project on IPRs and Sustainable Development, Genebra, ICTSD/UNCTAD, 2006. [Online]. Disponivel em: $<$ http://www.unctad.org/en/docs/iteipc20064 en.pdf $>$. Acesso em abr. 2007, p. v.
}

Revista Brasileira de Direito Internacional, Curitiba, v.7, n.7, jan./jun.2008 
[Les] Droits de propriété intellectuelle n'ont jamais été assez politique et économiquement importants ou controverses comme maintenant. Ils sont souvent mentionnés dans les discussions et débats sur les plus divers topiques, tels comme santé publique, sécurité alimentaire, éducation, commerce, politique industriel, savoirs traditionnels, biodiversité, biotechnologie, Internet, industrie d'amusement et média [notre traduction].

Malgré la croissance de l'intérêt manifesté quant aux savoirs traditionnels, le monde capitaliste a souvent regardé ces connaissances selon l'esprit sur lequel se fondent leurs lois de propriété intellectuelle : comme des savoirs qui appartiennent au domaine public, disponibles gratuitement pour être utilisés par quiconque. C'est telle interprétation qui a permis que certains savoirs traditionnels soient appropriés - en bonne forme juridique - par des entreprises de recherche et commerce, sans aucune compensation aux créateurs ou détenteurs du savoir. ${ }^{36}$

\section{a. LE SYSTÈME INTERNATIONAL DE PROTECTION ET LA POSSIBILITÉ DE PROTECTION PAR LUI-MÊME - RÈGLES DE L'OMC/ADPIC}

Les premières conventions internationales sur la propriété intellectuelle (Berne et Paris) ont été signées à la fin du XIXe. siècle. Elles étaient inspirées par des principes et des valeurs qui à l'époque se croyaient universaux. À ce temps-là, personne ne considérait que les savoirs autochtones pouvaient être utiles à n'importe quelle fin technologique, scientifique ou économique, et, pour cette raison, il n'existait pas une justification pour leur protection juridique

De nos jours, le système international de protection des droits de propriété intellectuelle est constitué dans le cadre de l'OMC, par I'ADPIC. II existe également plusieurs accords sur les différents aspects de la propriété intellectuelle au sein de l'Organisation mondiale de la propriété intellectuelle

\footnotetext{
${ }^{36}$ CORREA, Carlos Maria. Traditional knowledge and intellectual property: issues and options surrounding the protection of traditional knowledge - A discussion paper. Genebra: Quaker United Nations Office, 2001. [Online] Disponível em: www.iucn.org/themes/pbia/themes/trade/training/TK\%20and\%20Intellectual\%20Property.pdf. Acesso em nov. 2007, p.3.
}

Revista Brasileira de Direito Internacional, Curitiba, v.7, n.7, jan./jun.2008 
(OMPI) et aussi d'autres accords qui concernent le sujet au sein du système onusien, comme la CDB.

I L'OMPI

Dans le cadre de l'OMPI a été créé le Comité intergouvernemental sur savoirs traditionnels, ressources génétiques et expressions culturelles traditionnelles/folklore ${ }^{37}$. Le Comité discute des sujets concernant la protection des savoirs traditionnels. Dans le cadre du Comité ont été dispute divers sujets technique sur des aspects de cette protection :

- les conditions pour la divulgation de brevets relatifs aux savoirs traditionnels liés aux ressources génétiques;

- l'utilisation d'un système juridique sui generis pour la protection des savoirs traditionnels déterminé par leurs particularités;

- le développement des orientations, directives et clauses - pratiques contractuelles de propriété intellectuelle relatives à la distribution des profits aux communautés traditionnelles et l'accès aux ressources génétiques;

- la révision des critères en vigueur, car eux ne se appliquent pas aux savoirs traditionnels, et la nécessité de développer de nouveaux

\footnotetext{
37 "Le Comité intergouvernemental de la propriété intellectuelle relative aux ressources génétiques, aux savoirs traditionnels et au folklore de l'OMPI a été créé par l'Assemblée générale de l'Organisation en octobre 2000 (document WO/GA/26/6) en tant qu'instance internationale consacrée aux débats et au dialogue sur l'interaction de la propriété intellectuelle et des savoirs traditionnels, des ressources génétiques, et des expressions culturelles traditionnelles (folklore).

Le mandat actuel du comité (document WO/GA/30/8) demande qu' "il poursuive ...ses travaux sur les questions indiquées dans son mandat précédent" et stipule que "ses nouvelles activités seront notamment axées sur l'examen de la dimension internationale de ces questions, sans préjudice des travaux menées au sein d'autres instances, et aucun résultat de ses travaux n'est à exclure, y compris l'élaboration d'un ou plusieurs instruments internationaux". L'Assemblée générale a exhorté le comité intergouvernemental à accélérer ses travaux et a en outre prié le Bureau international de poursuivre son assistance au comité intergouvernemental en mettant à la disposition des États membres les compétences et la documentation nécessaires. » (ORGANISATION MONDIALE DE LA PROPRIÉTÉ INTELLECTUELLE. [Online] Disponível em: $<$ www.wipo.ints. Acesso em: nov. 2007).
}

Revista Brasileira de Direito Internacional, Curitiba, v.7, n.7, jan./jun.2008 
critères pour les savoirs traditionnels à fin de permettre les cataloguer, et;

- la création d'un guide pratique sur le sujet ${ }^{38}$.

Le Comité accorde aussi attention à l'établissement de registres et de bases de données des savoirs traditionnels qui peuvent être une protection préventive des savoirs traditionnels : si le savoir est dans la base de données il n'est pas nouveau, donc il est impossible de le breveter. Aussi la reconnaissance formelle des savoirs traditionnels par leur inscription dans la base de données peut-être considérée comme protection positive et une forme de droit sui generis. ${ }^{39}$ L'Inde et la Chine sont en train d'instaurer ces «bibliothèques numériques» en respectant les critères de la Classification internationale des brevets (CIB) de l'OMPI, qui permettent aux analystes des brevets par tout le monde d'accéder à ladite base de donnes et consulter si la demande de brevet qu'ils sont en train d'analyser découle ou non d'un savoir traditionnel, et si oui, ne pas octroyer le brevet.

En plus de la protection qui peut mettre fin ou peut, au moins, diminuer la biopiraterie, la documentation des savoirs traditionnels permet aussi de promouvoir l'exploitation durable de ces savoirs ${ }^{40}$ par les « occidentaux », ce qui représente la mise en œuvre du principe du développement durable.

Dans cette étude, le Comité est arrivé à la conclusion qu'il serait intéressant d'établir un traité relatif aux savoirs traditionnels. Pour la situation immédiate, le Comité suggère la création d'un mécanisme sui generis international de protection des savoirs traditionnels qui consisterait en l'adaptation des mécanismes existants de protection de propriété intellectuelle, avec l'accent sur les caractéristiques spécifiques des savoirs traditionnels.

\footnotetext{
${ }^{38}$ BAYLĀO, Raul S. \& BENSUSAN, Nurit. A questão das proteção dos conhecimentos tradicionais associados aos recurssos genéticos nos fóruns internacionais. In: BENSUSAN, Nurit \& LIMA, André (dir.). Quem Cala Consente? Subsídios para a proteção aos conhecimentos tradicionais. São Paulo: Instituto Socioambiental, 2003, pp. 18-19.

39 AUBERTIN, Catherine et al.. L'accès aux ressources génétiques et le partage des avantages : une question conflictuelle - exemples du Brésil et de la Bolivie. In : AUBERTIN, Catherine et al (dir.). Les marches de la biodiversité. Paris : IRD Éditions, 2007, p. 139.

${ }^{40}$ CORREA, Carlos Maria (dir.). Temas de derecho industrial y de la competencia: propiedad intelectual y políticas de desarrollo. Buenos Aires: Ciudad Argentina, 2005, pp. 216-218.
}

Revista Brasileira de Direito Internacional, Curitiba, v.7, n.7, jan./jun.2008 


\section{La CDB}

Sur le plan international, la CDB est le principal traité à protéger les savoirs traditionnels, qui font l'objet de son article 8(j) :

«Chaque Partie contractante, dans la mesure du possible et selon qu'il conviendra :

(...)

j) Sous réserve des dispositions de sa législation nationale, respecte, préserve et maintient les connaissances, innovations et pratiques des communautés autochtones et locales qui incarnent des modes de vie traditionnels présentant un intérêt pour la conservation et l'utilisation durable de la diversité biologique et en favorise l'application sur une plus grande échelle, avec l'accord et la participation des dépositaires de ces connaissances, innovations et pratiques et encourage le partage équitable des avantages découlant de l'utilisation de ces connaissances, innovations et pratiques; ${ }^{41}$ (nos soulignés).

C'est pour cette raison que la CDB ne protège pas seulement l'environnement, mais aussi les savoirs traditionnels ${ }^{42}$, qui sont une façon, déjà mentionnée, de protection et utilisation durable de la biodiversité. De ce fait, la CDB donne un cadre légal afin que l'utilisation des savoirs traditionnels ait lieu toujours de façon à respecter les objectifs de la Convention ${ }^{43}$.

La CDB est une convention-cadre dont la mise en œuvre revient aux États parties; c'est à eux de développer le cadre juridique pour la protection des savoirs traditionnels en donnant corps aux prescriptions de la Convention.

La CDB appelle les États-Parties, dans le cadre de l'exercice de leur souveraineté sur les ressources biologiques (article 3), à définir et reconnaître les droits des communautés autochtones et locales sur

\footnotetext{
${ }^{41}$ ORGANISATION DES NATIONS UNIES. Convention sur la diversité biologique. Rio de Janeiro, 5 junho 1992. [Online] Disponível em: <www.cbd.int $>$. Acesso em: jan. 2008.

${ }^{42}$ La CDB ne définit pas "savoirs traditionnels" (MÜLLER, Andréia N.. A proteçāo dos conhecimentos tradicionais por meio das indicações geográficas. In: RODRIGUES JR, Edson B.. \& POLIDO, Fabrício (dir.). Propriedade intelectual: novos paradigmas internacionais, conflitos e desafios. Rio de Janeiro: Elsevier, 2007, p. 322).

${ }^{43}$ Les objectifs de la CDB selon l'article 1er de la Convention «sont la conservation de la diversité biologique, l'utilisation durable de ses éléments et le partage juste et équitable des avantages découlant de l'exploitation des ressources génétiques, notamment grâce à un accès satisfaisant aux ressources génétiques et à un transfert approprié. Des techniques pertinentes, compte tenu de tous les droits sur ces ressources et aux techniques, et grâce à un financement adéquat. " (ORGANISATION DES NATIONS UNIES. Convention sur la diversité biologique. Rio de Janeiro, 5 junho 1992. [Online] Disponível em: <www.cbd.int>. Acesso em: jan. 2008).
}

Revista Brasileira de Direito Internacional, Curitiba, v.7, n.7, jan./jun.2008 
leurs savoirs et pratiques traditionnelles (article 8j) et les droits de propriété intellectuelle sur des technologies utilisant des ressources génétiques (article 16). ${ }^{44}$

Quand les États organisent la protection des savoirs traditionnels dans leur sphère juridique interne, ils doivent suivre les lignes établies par la Convention. Une de ces lignes peut être extraite de l'article 8(j): lorsqu'il mentionne l'utilisation à plus grande échelle du savoir traditionnel des autochtones, il pose aussi certaines conditions, comme l'accord et la participation des communautés autochtones dépositaires de ces connaissances dans ce processus ${ }^{45}$.

Afin de respecter cette condition, depuis l'entrée en vigueur de la CDB et la création d'un groupe de travail au sein de la Conférence des parties sur la mise en œuvre de l'article $8(j)^{46}$, a augmenté la participation des autochtones aux événements et à la prise de décisions sur le plan de la Convention ${ }^{47}$. Certains pays ont en effet intégré des autochtones à leur délégation nationale au groupe de travail ${ }^{48}$.

Cette condition se justifie, selon Aubertin et al. ${ }^{49}$, parce que la CDB se présente comme une tentative de réponse aux revendications des pays contre

${ }^{44}$ AUBERTIN, Catherine et al.. L'accès aux ressources génétiques et le partage des avantages : une question conflictuelle - exemples du Brésil et de la Bolivie. In : AUBERTIN, Catherine et al (dir.). Les marches de la biodiversité. Paris : IRD Éditions, 2007, pp. 135-136.

${ }_{45}$ ARBOUR, Jean-Maurice \& LAVALLÉE, Sophie.Droit international de l'environnement, Cowansville : Éditions Yvons Blais/Bruylant, p. 479.

${ }^{46}$ Le Groupe de travail sur l'article 8(j) était créé en 1998 par la 4ème Conférence de parties (COP4) de la CDB. À la 5ème Conférence de parties (COP5) de la CDB, en 2000, il a établi un programme de travail pour mettre en œuvre les dispositions de l'article 8(j), ce qui a possibilité la participation de peuples autochtones dans l'obtention des objectifs de la Convention. Le programme de travail du Groupe pour la biennie 2006-2008 inclut le développement d'éléments pour un système sui generis de protection des savoirs traditionnels, pour que les deux organismes (CDB/COPs-OMPI) travaillent en coopération sur ce sujet, afin de créer une synergie, et non une duplicité de travail (COP 5, Programme of work on the implementation of article $8(j)$ and related provisions of the convention on biological diversity -IV, Ways and means).

${ }^{47}$ Mais les objectifs établis dans le préambule de la Décision V/16 du COP 5 envisage aussi une augmentation de la participation des autochtones aux plans régional, national et international général, non seulement dans le cadre de la CDB (COP 5. Programme of work on the implementation of article 8(j) and related provisions of the convention on biological diversity -IV, Ways and means. Décision V/16. Nairobi, 26 maio 2000. [Online] Disponível em: $<$ http://www.cbd.int/decisions/?m=COP-05\&id=7158\&lg=0 >. Acesso em abr. 2008).

${ }_{48}$ ARBOUR, Jean-Maurice \& LAVALLÉE, Sophie.Droit international de l'environnement, Cowansville : Éditions Yvons Blais/Bruylant, 2006, p. 480.

${ }^{49}$ AUBERTIN, Catherine et al (dir.). Les marches de la biodiversité. Paris : IRD Éditions, 2007, pp. 85-86.

Revista Brasileira de Direito Internacional, Curitiba, v.7, n.7, jan./jun.2008 
un pillage du Sud par des industries et des chercheurs du Nord qui auraient breveté dans leurs pays de prétendues découvertes, tirées de ressources ou dérivant de savoirs traditionnels, à l'insu des communautés ou pays du Sud qui les ont fournis, et sans que ceux-ci soient associés aux avantages. C'est cela la biopiraterie.

\section{L'ADPIC}

L'ADPIC ne fait pas mention des savoirs traditionnels. Toutefois, par le biais du Paragraphe 19 de la Déclaration ministérielle de Doha ${ }^{50}$, les États membres de l'OMC ont souligné le besoin que le Conseil des ADPIC travaille pour la protection des savoirs traditionnels ${ }^{51}$.

Les discussions au sein du Conseil des ADPIC ont pris le chemin de la possibilité de protection des savoirs traditionnels par le biais d'une extension de la protection par l'indication géographique accordée aujourd'hui aux vins et aux spiritueux. "Les indications géographiques sont des droits collectifs que protègent la réputation et non l'innovation ${ }^{52}$ donc applicable aux savoirs traditionnels parce qu'ils ne sont pas d'invention ou de découverte, et dans certains cas ne sont pas susceptibles d'application industrielle non plus ${ }^{53}$. Les indications géographiques sont aussi utiles aux savoirs traditionnels parce qu'elles sont illimitées dans le temps et inaliénables. L'extension des indications géographiques aux savoirs traditionnels est défendue parce qu'elle encourage les producteurs à commercialiser leurs produits dans le marché international, en promouvant le commerce international.

Les avantages de cette façon de protéger les savoirs consistent aussi dans le fait que le droit est accordé à un groupe, et non à un individu, et que le

50 ORGANISATION MONDIALE DU COMMERCE. Déclaration ministérielle. WT/MIN(01)/DEC/1. Doha, 20 novembro 2001. [Online] Disponível em: < <www.wto.org>. Acesso em: abr. 2008.

${ }^{51}$ CORREA, Carlos Maria (dir.). Temas de derecho industrial y de la competencia: propiedad intelectual y políticas de desarrollo. Buenos Aires: Ciudad Argentina, 2005, p. 205.

52 BOISVERT, Valérie \& CARON, Armelle. Valorisation économique des ressources et nouveaux marchés. In : AUBERTIN, Catherine et al (dir.). Les marches de la biodiversité. Paris: IRD Éditions, 2007, 195.

${ }^{53}$ Les indications géographiques sont objet des articles 22 à 24 de l'ADPIC.

Revista Brasileira de Direito Internacional, Curitiba, v.7, n.7, jan./jun.2008 
droit de propriété intellectuelle, ainsi comme le savoir, peut être transfère de génération à génération ${ }^{54}$.

Néanmoins, comme I'ADPIC établit seulement des niveaux minimaux de protection des droits de propriété intellectuelle, rien n'empêche ses signataires d'augmenter les niveaux de protection dans la sphère nationale, selon l'article 1 de l'ADPIC ${ }^{55}$. Cela veut dire que l'Accord permet la protection nationale des savoirs traditionnels par le biais de droits de propriété intellectuelle ${ }^{56}$.

$\mathrm{Au}$ sein de I'ADPIC se déroule une discussion entre les pays développés qui ont des intérêts aux savoirs traditionnels et les pays en développement (PED) qui abritent la plus grande partie des populations avec des savoirs traditionnels. La polémique tourne autour du statut des savoirs traditionnels en face du droit de propriété intellectuelle. Les pays développés disent que, comme l'ADPIC ne les mentionne pas la protection des savoirs traditionnels par les biais des droits de propriété intellectuelle, elle n'existe pas. Par contre, les pays non développés disent qu'il faut respecter la CDB. Mais en effet, l'ADPIC et la CDB sont complémentaires et doivent être interprétés et appliqués conjointement ${ }^{57}$, cela fait dire, impose le respect aux conditions imposés par la CDB à fin de octroi les droits prévues par l'ADPIC.

\footnotetext{
${ }^{54}$ MÜLLER, Andréia N.. A proteçāo dos conhecimentos tradicionais por meio das indicações geográficas. In: RODRIGUES JR, Edson B.. \& POLIDO, Fabrício (dir.). Propriedade intelectual: novos paradigmas internacionais, conflitos e desafios. Rio de Janeiro: Elsevier, 2007, pp. 322323.

${ }^{55}$ Article premier

Nature et portée des obligations

1. Les Membres donneront effet aux dispositions du présent accord. Les Membres pourront, sans que cela soit une obligation, mettre en œuvre dans leur législation une protection plus large que ne le prescrit le présent accord, à condition que cette protection ne contrevienne pas aux dispositions dudit accord. Les Membres seront libres de déterminer la méthode appropriée pour mettre en œuvre les dispositions du présent accord dans le cadre de leurs propres systèmes et pratiques juridiques.

${ }^{56}$ ZAZZALI, Jorge C.. Propiedad intelectual, diversidad biológica y conocimentos tradicionales una visión desde los Andes y la Amazonia. In: CORREA, Carlos Maria (dir.). Temas de derecho industrial y de la competencia: biotecnología y derecho. Buenos Aires: Ciudad Argentina, 1997, p. 123.

${ }^{57}$ ZAZZALI, Jorge C.. Propiedad intelectual, diversidad biológica y conocimentos tradicionales una visión desde los Andes y la Amazonia. In: CORREA, Carlos Maria (dir.). Temas de derecho industrial y de la competencia: biotecnología y derecho. Buenos Aires: Ciudad Argentina, 1997, p. 126.
}

Revista Brasileira de Direito Internacional, Curitiba, v.7, n.7, jan./jun.2008 
IV La Possibilité De Protection Par Le Système Existant - Règles De L'OMC/ADPIC

Afin de défendre les savoirs traditionnels, les pays où se trouvent les communautés détentrices de ces savoirs peuvent tirer parti des dispositions existantes dans les domaines du droit international de l'environnement et celui de la propriété intellectuelle.

II est possible d'adapter les modèles actuels de protection des droits de propriété intellectuelle établis par l'ADPIC pour protéger les savoirs traditionnels comme Correa ${ }^{58}$ l'a démontré :

Le droit d'auteur (articles 9 à 14 de l'ADPIC) peut être utilisé pour protéger les manifestations artistiques des détenteurs du savoir traditionnel contre la reproduction non autorisée et contre l'exploitation ${ }^{59}$.

Les brevets (articles 27 à 34 de l'ADPIC) peuvent être utilisés pour protéger les solutions techniques qui trouvent une applicabilité dans l'industrie et qui sont inédites et inventives, et aussi pour les processus d'obtention et exploitation des ressources biogénétiques, pourvu qu'ils observent les exigences pour la concession de brevet comme les techniques non divulgués.

Le dessin industriel (articles 25 et 26 de l'ADPIC) peut être appliqué à la protection du dessin des outils, des meubles et des articles de poterie et en cuir.

Les indications géographiques et les dénominations d'origine (articles 22 à 24 de l'ADPIC), en plus de ce qui a était mentionné antérieurement peuvent ajouter valeur aux produits autochtones fabriqués dans une région déterminée selon un processus découlant d'un savoir traditionnel.

\footnotetext{
${ }^{58}$ CORREA, Carlos Maria. Traditional knowledge and intellectual property: issues and options surrounding the protection of traditional knowledge - A discussion paper. Genebra: Quaker United Nations Office, 2001. [Online] Disponível em: www.iucn.org/themes/pbia/themes/trade/training/TK\%20and\%20Intellectual\%20Property.pdf. Acesso em nov. 2007, p. 11.

${ }^{59}$ Le Canada protège par le biais de droit d'auteur (Loi sur le droit d'auteur) les créations fondées sur la tradition autochtone, comme les masques, les totems et les enregistrements sonores (CORREA, Carlos Maria (dir.). Temas de derecho industrial y de la competencia: propiedad intelectual y políticas de desarrollo. Buenos Aires: Ciudad Argentina, 2005, pp. 209210).
}

Revista Brasileira de Direito Internacional, Curitiba, v.7, n.7, jan./jun.2008 
La protection des renseignements non divulgués (article 39 de l'ADPIC ${ }^{60}$ ) peut être utilisée pour protéger les savoirs traditionnels pas encore divulgués qui peuvent avoir une valeur technologique et économique.

La protection pour les marques ${ }^{61}$ (articles 15 à 21 de l'ADPIC) ${ }^{62}$ apporte le grand avantage ${ }^{63}$ d'être une protection illimitée dans le temps ${ }^{64}$ et la possibilité de posséder le droit collectivement. Cette protection peut être aussi utilisée dans la protection des signes et de symboles.

Un avantage d'adapter les règles existantes c'est que cela coûterait moins cher que créer un nouveau système. ${ }^{65}$ Par contre, ceux qui sont contre

${ }^{60}$ Article 39

1. En assurant une protection effective contre la concurrence déloyale conformément à l'article 10bis de la Convention de Paris (1967), les Membres protégeront les renseignements non divulgués conformément au paragraphe 2 et les données communiquées aux pouvoirs publics ou à leurs organismes conformément au paragraphe 3.

2. Les personnes physiques et morales auront la possibilité d'empêcher que des renseignements licitement sous leur contrôle ne soient divulgués à des tiers ou acquis ou utilisés par eux sans leur consentement et d'une manière contraire aux usages commerciaux honnêtes (Aux fins de cette disposition, l'expression "d'une manière contraire aux usages commerciaux honnêtes" s'entendra au moins des pratiques telles que la rupture de contrat, l'abus de confiance et l'incitation au délit, et comprend l'acquisition de renseignements non divulgué s par des tiers qui savaient que ladite acquisition impliquait de telles pratiques ou qui ont fait preuve d'une grave négligence en l'ignorant.), sous réserve que ces renseignements:

a) soient secrets en ce sens que, dans leur globalité ou dans la configuration et l'assemblage exacts de leurs éléments, ils ne sont pas généralement connus de personnes appartenant aux milieux qui s'occupent normalement du genre de renseignements en question ou ne leur sont pas aisément accessibles;

b) aient une valeur commerciale parce qu'ils sont secrets; et

c) aient fait l'objet, de la part de la personne qui en a licitement le contrôle, de dispositions raisonnables, compte tenu des circonstances, destinées à les garder secrets.

3. Lorsqu'ils subordonnent l'approbation de la commercialisation de produits pharmaceutiques ou de produits chimiques pour l'agriculture qui comportent des entités chimiques nouvelles à la communication de données non divulguées résultant d'essais ou d'autres données non divulguées, dont l'établissement demande un effort considérable, les Membres protégeront ces données contre l'exploitation déloyale dans le commerce. En outre, les Membres protégeront ces donné es contre la divulgation, sauf si cela est nécessaire pour protéger le public, ou à moins que des mesures ne soient prises pour s'assurer que les données sont protégées contre l'exploitation déloyale dans le commerce.

${ }^{61}$ Selon Barbosa «la marque est le signé visuellement représente, qui est configure au fin spécifique de distinguer l'origine des produits et services " [notre traduction]. (BARBOSA, Denis B..Uma introduçāo à propriedade intelectual. Rio de Janeiro: Lumen Juris, 2003, p. 803).

${ }^{62}$ Cette possibilité est apportée par Aubertin et al. (AUBERTIN, Catherine et al (dir.). Les marches de la biodiversité. Paris : IRD Éditions, 2007, p. 160).

${ }^{63}$ CORREA, Carlos Maria (dir.). Temas de derecho industrial y de la competencia: propiedad intelectual y políticas de desarrollo. Buenos Aires: Ciudad Argentina, 2005, p. 210.

${ }^{64}$ Même s'il y a un besoin de renouvellement de temps en temps.

${ }^{65}$ MÜLLER, Andréia N.. A proteçāo dos conhecimentos tradicionais por meio das indicações geográficas. In: RODRIGUES JR, Edson B.. \& POLIDO, Fabrício (dir.). Propriedade intelectual: novos paradigmas internacionais, conflitos e desafios. Rio de Janeiro: Elsevier, 2007, p. 329.

Revista Brasileira de Direito Internacional, Curitiba, v.7, n.7, jan./jun.2008 
ces options, comme quelques ONG internationales ${ }^{66}$, considèrent qu'il y a une incompatibilité entre les concepts capitalistes des droits de propriété intellectuelle et les pratiques et valeurs philosophiques des autochtones. Dans le cas de brevets spécifiquement, ils craignent que le caractère exclusif de propriété conférée par les brevets puisse priver les populations détentrices des savoirs de leurs usages traditionnels ${ }^{67}$.

\subsection{LA POSSIBILITÉ DE PROTECTION PAR UN SYSTÈME SUI} GENERIS ${ }^{68}$

Une partie des pays du Sud éprouvent des difficultés techniques et économiques à se doter de systèmes de protection de savoirs traditionnels conformément à l'article $15^{69}$ de la $\mathrm{CDB}^{70}$; d'autres pays ont déjà réussi ou sont déjà en bonne route.

\footnotetext{
${ }^{66}$ Aubertin et Moretti mentionnent Third World Network, RAFI/ETC Group, Genetic Resources Action International (GRAIN) et Research Foundation for Science, Technology and Natural Resources Policy (AUBERTIN, Catherine \& MORETTI, Christian. La biopiraterie entre illégalité et illégitimité. In : AUBERTIN, Catherine et al (dir.). Les marches de la biodiversité. Paris: IRD Éditions, 2007, p. 99).

${ }^{67}$ AUBERTIN, Catherine \& MORETTI, Christian. La biopiraterie entre illégalité et illégitimité. In : AUBERTIN, Catherine et al (dir.). Les marches de la biodiversité. Paris: IRD Éditions, 2007, 100.

${ }^{68}$ Un système sui generis est un système différent des existants, un nouveau système.

${ }^{69}$ Article 15. Accès aux ressources génétiques

1. Étant donné que les États ont droit de souveraineté sur leurs ressources naturelles, le pouvoir de déterminer l'accès aux ressources génétiques appartient aux gouvernements et est régi par la législation nationale.

2. Chaque Partie contractante s'efforce de créer les conditions propres à faciliter l'accès aux ressources génétiques aux fins d'utilisation écologiquement rationnelle par d'autres Parties contractantes et de ne pas imposer de restrictions allant à l'encontre des objectifs de la présente Convention.

3. Aux fins de la présente Convention, on entend par ressources génétiques fournies par une Partie contractante, et dont il est fait mention dans le présent article et aux articles 16 et 19 ciaprès, exclusivement les ressources qui sont fournies par des Parties contractantes qui sont des pays d'origine de ces ressources ou par des Parties qui les ont acquises conformément à la présente Convention.

4. L'accès, lorsqu'il est accordé, est régi par des conditions convenues d'un commun accord et est soumis aux dispositions du présent article.

5. L'accès aux ressources génétiques est soumis au consentement préalable donné en connaissance de cause de la Partie contractante qui fournit lesdites ressources, sauf décision contraire de cette Partie.

6. Chaque Partie contractante s'efforce de développer et d'effectuer des recherches scientifiques fondées sur les ressources génétiques fournies par d'autres Parties contractantes avec la pleine participation de ces Parties et, dans la mesure du possible, sur leur territoire.
}

Revista Brasileira de Direito Internacional, Curitiba, v.7, n.7, jan./jun.2008 
II y a quelques systèmes sui generis dans la sphère nationale, qui adoptent des dispositions législatives reconnaissant aux communautés autochtones un droit de participation à la prise de décision quant à l'utilisation du savoir et des pratiques autochtones concernant des ressources biologiques. Ce typé de système a été adopté par le droit interne en quelques pays, parmi lesquels on compte le Costa Rica, l'Équateur, la Malaisie, le Pérou, les Philippines et la Thaïlande.

La Thaïlande a créé un régime spécial pour la médecine traditionnelle ${ }^{71}$ par lequel quelques formules, qui font partie du savoir traditionnel, ont le gouvernement comme titulaire et destinataire des profits; d'autres ont les communautés qui les ont créées, et d'autres encore appartiennent au domaine public. $^{72}$

La loi péruvienne ${ }^{73}$ "prévoit aussi que les utilisateurs commerciaux et industriels des savoirs autochtones doivent revendiquer une licence qui prend la forme d'un contrat écrit avec les détenteurs dudit savoir ${ }^{74}$. Ce contrat est rédigé en espagnol et dans l'idiome autochtone. Les informations du contrat sont envoyées aux principaux bureaux de brevets du monde pour qu'ils soient consultés lors de l'analyse des critères de nouveauté et inévitabilité à fin

7. Chaque Partie contractante prend les mesures législatives, administratives ou de politique générale appropriées, conformément aux articles 16 et 19 et, le cas échéant, par le biais du mécanisme de financement créé en vertu des articles 20 et 21, pour assurer le partage juste et équitable des résultats de la recherche et de la mise en valeur ainsi que des avantages résultant de l'utilisation commerciale et autre des ressources génétiques avec la Partie contractante qui fournit ces ressources. Ce partage s'effectue selon des modalités mutuellement convenues (CDB, supra note 1).

${ }^{70}$ AUBERTIN, Catherine et al (dir.). Les marches de la biodiversité. Paris : IRD Éditions, 2007, p. 18.

71 THAILAND. Act on Protection and Promotion of Traditional Thai Medicinal Intelligence, B.E 2542 ('Thai Act') apud ORGANISATION MONDIALE DE LA PROPRIÉTÉ INTELLECTUELLE. Intergovernmental committee on intellectual property and genetic resources, traditional knowledge and folklore, The Protection of TRADITIONAL KNOW Mechanisms Document prepared by the Secretariat LEDGE: Revised Outline of Policy Options and Legal. WIPO/GRTKF/IC/9/INF/5. Genebra, 27 março 2006. [Online] Disponível em: <http://www.wipo.int/edocs/mdocs/tk/en/wipo_grtkf_ic_9/wipo_grtkf_ic_9_inf_5.doc>. Acesso em: abr. 2008.

72 Toutes les formules, de n'importe quelle catégorie, peuvent être utilisées de façon non commerciale gratuitement, à une quantité limitée, par les autochtones dans leurs communautés.

${ }^{73}$ Propuesta de régimen de protección de los conocimientos colectivos de los pueblos y comunidades indígenas vinculados a los recursos biológicos, adopté en 2002.

74 ARBOUR, Jean-Maurice \& LAVALLÉE, Sophie. Droit international de l'environnement, Cowansville : Éditions Yvons Blais/Bruylant, 2006, p. 481.

Revista Brasileira de Direito Internacional, Curitiba, v.7, n.7, jan./jun.2008 
d'éviter que quelqu'un reçoive un brevet d'un savoir autochtone enregistré au Pérou. La loi sépare aussi les savoirs qui se trouvent déjà dans le domaine public et ceux qui sont confidentiels. Finalement, la loi détermine que l'argent reçu de ces contrats soit destiné à un fond pour le développement des peuples autochtones $^{75}$.

Comme la majorité des communautés autochtones n'ont pas les mêmes valeurs du monde capitaliste - parfois elles ne sont même pas familiarisées avec les concepts les plus élémentaires du capitalisme, tels que l'argent et le paiement —, il faudra que le capitalisme s'adapte à ces conditions locales et qu'il paye pour le savoir traditionnel directement à l'État où se trouve la communauté, qui s'occupera de gérer l'argent à leur bénéfice (comme dans le cas péruvien). D'autres fois, le payement peut se faire sous la forme de (a) versements de redevances, qui peuvent être toujours gérées par une tierce partie autre que non la communauté; (b) de la copropriété des droits de propriété intellectuelle; ou encore (c) de l'accès gratuit aux technologies et aux produits obtenus à partir des savoirs traditionnels.

Dans l'élaboration d'un système sui generis, il faut toujours définir la nature des droits conférés, s'ils sont exclusifs ou non, vu que c'est possible de considérer la concession du droit à la rémunération sans l'exercice d'un droit d'exclusivité. C'est-à-dire, un régime contre les appropriations sans consentement des savoirs traditionnels, mais sans que quelque sorte de droit d'exclusivité soit octroyé à celui qui utilise ou veut utiliser le savoir commercialement, car cela peut être contraire aux valeurs autochtones.

Le Panama $^{76}$ a établi un régime spécial de registre de propriété intellectuelle pour la protection des savoirs traditionnels qui peuvent être associés à l'identité culturelle d'une communauté et peuvent être

75 SILVA, Letícia B.. É possível negociar a biodiversidade? Conhecimentos tradicionais, propriedade intelectual e biopirataria. In: BARRAL, Welber \& PIMENTEL, Luiz Otavio (dir.). Propriedade Intelectual e desenvolvimento. Florianópolis: Fundação Boiteux, 2006, p. 320.

${ }^{76}$ Par le biais de la Loi $n^{\circ} 20$ du 26 juillet 2000 , qui est entré en vigueur le 27 juin 2000 , et a été promulguée dans la Gazette Officielle No. 24.083 réglée par le Décret Exécutive No. 12, du 20 mars 2001, intitulé Special Intellectual Regime Governing the Collective Rights of Indigenous Peoples, for the Protection and Defense of their Cultural Identity and their Traditional Knowledge, and Other Provisions.(ORGANISASTION MONDIALE DE LA PROPRIÉTÉ INTELLECTUELLE. Survey on Existing Forms of Intellectual Property Protection for Traditional Knowledge. Document WIPO/GRTKF/IC/2/5. [Online] Disponível em: <www.wipo.int>. Acesso em: abr. 2008).

Revista Brasileira de Direito Internacional, Curitiba, v.7, n.7, jan./jun.2008 
commercialisés ${ }^{77}$. Le régime panaméen interdit la protection de l'héritage culturel autochtone (y inclus les savoirs traditionnels) par des droits d'exclusivité du système de propriété intellectuelle, tels que droit d'auteur, indication géographique, dessin industriel et marques, sauf quand la demande de protection est faite par les autochtones eux-mêmes. Donc, les autochtones peuvent être détenteurs de droits de propriété intellectuelle sur leur héritage culturel, et eux peuvent licencier leurs droits de propriété intellectuelle à des tiers, et ces droits ne peuvent pas être objet d'une licence obligatoire ${ }^{78}$.

Au Brésil, le projet de loi qui réglemente l'accès aux ressources génétiques inclut sous sa protection les savoirs traditionnels associés définis comme «information ou pratique individuelle ou collective des communautés indigènes ou locales ayant une valeur réelle ou potentielle associée au patrimoine génétique ${ }^{79}$. Dans cette proposition, les savoirs traditionnels sont tenus comme partie du patrimoine culturel brésilien. Ces savoirs pourront être reconnus comme propriété collective d'une communauté ${ }^{80}$, ce qui peut les rendre disponibles à des tiers moyennant son consentement préalable.

Dans le cas de la création d'un système international sui generis, Correa $^{81}$ considère qu'il peut être un seul système pour toutes les formes de savoir traditionnel ou bien des systèmes différents pour: (a) la création artistique et le folklore; (b) savoirs associés aux ressources génétiques utilisés à l'industrie alimentaire et à l'agriculture; et (c) médecine traditionnelle. En tout cas, ce système (ou ces systèmes) devra principalement prévoir la possibilité

\footnotetext{
${ }^{77}$ MÜLLER, Andréia N.. A proteçāo dos conhecimentos tradicionais por meio das indicações geográficas. In: RODRIGUES JR, Edson B.. \& POLIDO, Fabrício (dir.). Propriedade intelectual: novos paradigmas internacionais, conflitos e desafios. Rio de Janeiro: Elsevier, 2007, p. 314.

${ }^{78}$ ORGANISATION MONDIALE DE LA PROPRIÉTÉ INTELLECTUELLE. Intergovernmental committee on intellectual property and genetic resources, traditional knowledge and folklore, The Protection of TRADITIONAL KNOW Mechanisms Document prepared by the Secretariat LEDGE: Revised Outline of Policy Options and Legal. WIPO/GRTKF/IC/9/INF/5. Genebra, 27 março $2006 . \quad$ [Online] Disponível em: $<$ http://www.wipo.int/edocs/mdocs/tk/en/wipo_grtkf_ic_9/wipo_grtkf_ic_9_inf_5.doc>. Acesso em: abr. 2008.

${ }^{79}$ AUBERTIN, Catherine et al. L'accès aux ressources génétiques et le partage des avantages : une question conflictuelle - exemples du Brésil et de la Bolivie. In : AUBERTIN, Catherine et al (dir.). Les marches de la biodiversité. Paris : IRD Éditions, 2007, pp. 131-134.

${ }^{80}$ AUBERTIN, Catherine et al. L'accès aux ressources génétiques et le partage des avantages : une question conflictuelle - exemples du Brésil et de la Bolivie. In : AUBERTIN, Catherine et al (dir.). Les marches de la biodiversité. Paris: IRD Éditions, 2007, pp. 138-139.

${ }^{81}$ CORREA, Carlos Maria (dir.). Temas de derecho industrial y de la competencia: propiedad intelectual y políticas de desarrollo. Buenos Aires: Ciudad Argentina, 2005, p. 14.
}

Revista Brasileira de Direito Internacional, Curitiba, v.7, n.7, jan./jun.2008 
de propriété collective (par une ou plusieurs communautés) d'un dit savoir et définir la nature du droit confère, s'il sera exclusif ou non. Le système devra aussi, selon Zazzali ${ }^{82}$ :

(a) Identifier les communautés qui ont propriété collective des savoirs traditionnels;

(b) Permettre que ces communautés régissent l'usage commercial de leurs savoirs traditionnels, par le biais du consentement préalable;

(c) Établir l'inaliénabilité de cette propriété collective;

(d) Empêcher les concessions d'autres droits de propriété intellectuelle qui découlent de ces savoirs traditionnels;

(e) Créer des mécanismes que garantit le partage équitable des avantages découlant de l'exploitation commerciale des dits savoirs;

(f) Compter sur la participation des communautés autochtones dans le développement du système lui-même.

II n'existe pas encore un système sui generis sur le plan international. Pour Correa ${ }^{83}$, un système international sui generis peut être trop spécifique et peu flexible pour l'adaptation aux besoins locaux. En effet, le plus grand problème pour la création d'un système international sui generis c'est qu'il intéresse les pays du Sud, mais non les pays du Nord; il sera donc très improbable que ces derniers signent l'accord dans le cadre da l'OMPI, de la CDB (qui jusqu'à aujourd'hui n'a pas été elle-même signée par les États-Unis) ou indépendamment de quelque forum déjà existant. Quelle sera l'effectivité de cet accord signé seulement par des pays du Sud? Peut-être non plus que créer une loi modèle pour être implantée sur le plan national des pays du Sud que n'ont pas encore le fait. Même si les pays du Nord signent l'accord, signeront-ils

\footnotetext{
${ }^{82}$ ZAZZALI, Jorge C.. Propiedad intelectual, diversidad biológica y conocimentos tradicionales una visión desde los Andes y la Amazonia. In: CORREA, Carlos Maria (dir.). Temas de derecho industrial y de la competencia: biotecnología y derecho. Buenos Aires: Ciudad Argentina, 1997, pp. 125-126.

${ }^{83}$ CORREA, Carlos Maria (dir.). Temas de derecho industrial y de la competencia: propiedad intelectual y políticas de desarrollo. Buenos Aires: Ciudad Argentina, 2005, p.214.
}

Revista Brasileira de Direito Internacional, Curitiba, v.7, n.7, jan./jun.2008 
un accord qui contienne des mesures vraiment contraignantes qui puissent être de facto effectives?

\subsection{LA POSSIBILITÉ DE PROTECTION PAR LES CLAUSES ADPIC-} EXTRA

Une autre option possible, dans le cadre international, c'est l'inclusion des clauses de protection des droits de propriété intellectuelle des savoirs traditionnels dans les Accords régionaux de commerce et dans les Accords bilatéraux de commerce, comme clauses ADPIC-extra ${ }^{84}$.

Dans ce cas, l'inclusion de ces clauses peut avoir un impact généralisé dans le scénario international et vraiment bon particulièrement pour les PED : en face du traitement de la nation la plus favorisée, ces clauses s'appliqueront à touts le pays membre de l'OMC, une fois que ces accords bilatéraux ne font pas partie des exceptions prévues à l'article $4^{85}$ de l'ADPIC et n'ont non plus prévision des exceptions eux-mêmes. L'adoption des clauses ADPIC-extra garantirait en plus que ces mesures auront des effets dans un cadre contraignant, effectif, qui embrassera et obligera presque tous les pays du Nord. Dans le cas du système sui generis international, par contre, la participation des pays du Nord, l'effectivité et la contrainte sont de questions en ouvert jusqu'à présent.

\footnotetext{
${ }^{84}$ Les clauses ADPIC-extra régissent les sujets qui ne sont pas réglés par cet Accord.

${ }^{85}$ Article 4

Traitement de la nation la plus favorisée

En ce qui concerne la protection de la propriété intellectuelle, tous avantages, faveurs, privilèges ou immunités accordés par un Membre aux ressortissants de tout autre pays seront, immédiatement et sans condition, étendus aux ressortissants de tous les autres Membres. Sont exemptés de cette obligation tous les avantages, faveurs, privilèges ou immunités accordés par un Membre:

a) qui découlent d'accords internationaux concernant l'entraide judiciaire ou l'exécution des lois en général et ne se limitent pas en particulier à la protection de la propriété intellectuelle;

b) qui sont accordés conformément aux dispositions de la Convention de Berne (1971) ou de la Convention de Rome qui autorisent que le traitement accordé soit fonction non pas du traitement national mais du traitement accordé dans un autre pays;

c) pour ce qui est des droits des artistes interprètes ou exécutants, des producteurs de phonogrammes et des organismes de radiodiffusion qui ne sont pas visés par le présent accord;

d) qui découlent d'accords internationaux se rapportant à la protection de la propriété intellectuelle dont l'entrée en vigueur précède celle de l'Accord sur l'OMC, à condition que ces accords soient notifiés au Conseil des ADPIC et ne constituent pas une discrimination arbitraire ou injustifiable à l'égard des ressortissants d'autres Membres.
}

Revista Brasileira de Direito Internacional, Curitiba, v.7, n.7, jan./jun.2008 


\section{CONCLUSION}

Arbour et Lavallée ${ }^{86}$ rapportent que, jusqu'à présent, ce sont très rares les cas de communautés autochtones, principalement dans les PED, qui ont reçu des compensations à cause de l'utilisation de leurs connaissances dans l'élaboration de produits par les industries occidentales. Partant, la protection de la propriété intellectuelle du savoir traditionnel est urgente, afin de garantir ces droits.

D'après Sachs, des lois de protection aux droits de propriété intellectuelle qui ne sont pas bien faites finissent par empêcher «le monde du bas revenu de monter l'escalier du développement ${ }^{87}$. Et pour que la réglementation soit effective, il est nécessaire que les organismes internationaux qui participent au processus aident techniquement les PED dans la mise en œuvre de la réglementation et aussi qu'ils apprennent aux communautés autochtones à s'en utiliser. C'est le cas des institutions comme la Banque Mondiale, le Fond monétaire international, l'OMC et l'ONU, principalement par le biais de l'UNCTAD. En 2000, cette dernière a commencé un projet sur les savoirs traditionnels, avec la promotion d'une rencontre d'experts sur les expériences nationales et les systèmes de protection aux savoirs traditionnels et les innovations. Cette réunion a produit le Rapport de la réunion d'experts sur les systèmes et l'expérience des pays en matière de protection des connaissances traditionnelles, de l'innovation et des pratiques ${ }^{88}$.

Pour protéger et promouvoir les savoirs traditionnels, il faut mettre en place ces conditions : le contrôle sur l'usage rationnel des ressources naturelles et le respect pour les valeurs autochtones, matérialisées dans une législation

\footnotetext{
${ }^{86}$ ARBOUR, Jean-Maurice \& LAVALLÉE, Sophie.Droit international de l'environnement, Cowansville: Éditions Yvons Blais/Bruylant, 2006, pp. 482-483.

${ }^{87}$ SACHS, Jeffrey. O fim da pobreza. São Paulo: Companhia das Letras, 2005, p. 51.

${ }^{88}$ CONFÉRENCE DES NATIONS UNIES SUR LE COMMERCE ET LE DÉVELOPPEMENT. Rapport de la réunion d'experts sur les systèmes et l'expérience des pays en matière de protection des connaissances traditionnelles, de l'innovation et des pratiques. TD/B/COM.1/33; TD/B/COM.1/EM.13/3. Genebra, 7 dezembro 2000. [Online] Disponível em: <www.unctad.org>. Acesso em abr. 2008.
}

Revista Brasileira de Direito Internacional, Curitiba, v.7, n.7, jan./jun.2008 
appropriée qui doit inclure la protection par le biais des droits de propriété intellectuelle. ${ }^{89}$

De cette façon, les droits de propriété intellectuelle - si chers au capitalisme global - travailleront pour les valeurs des communautés autochtones.

Quoique soit le modèle de protection des droits de propriété intellectuelle des savoirs traditionnels adopté parmi ceux considérés ici, le fait qu'il ait une réglementation de ce sujet promouvra non seulement la valorisation économique des savoirs traditionnels, mais aussi la valorisation de la diversité culturelle et de droits politiques des communautés autochtones, en contribuant à garantir leur souveraineté territoriale et le contrôle sur leurs ressources ${ }^{90}$, et finalement un développement durable pour eux et pour tout le monde.

${ }^{89}$ PROGRAMME DES NATIONS UNIES POUR L'ENVIRONNEMENT/CONVENTION SUR LA DIVERSITÉ BIOLOGIQUE Traditional knowledge and biological diversity: Note by the Executive Secretary. UNEP/CBD/TKBD/1/2. Madrid, 18 outubro 1997. [Online] Disponível em: $<$ http://www.cbd.int/doc/meetings/tk/wstkbd-01/official/wstkbd-01-02-en.pdf>. Acesso em: abr. 2008.

90 BOISVERT, Valérie \& VIVIEN, Franck-Dominique. Un marché pour la biodiversité. In: AUBERTIN, Catherine et al. (dir.). Les marches de la biodiversité. Paris : IRD Éditions, 2007, p. 242.

Revista Brasileira de Direito Internacional, Curitiba, v.7, n.7, jan./jun.2008 


\section{RÉFÉRENCES}

ABBOTT, Frederick M.. Intellectual property provisions of bilateral and regional trade agreements in light of U.S. federal law. In: UNCTAD-ICTSD Project on IPRs and Sustainable Development, Genebra, ICTSD/UNCTAD, 2006. [Online]. Disponivel em: <http://www.unctad.org/en/docs/iteipc20064_en.pdf>. Acesso em abr. 2007.

ARBOUR, Jean-Maurice \& LAVALLÉE, Sophie.Droit international de l'environnement, Cowansville: Éditions Yvons Blais/Bruylant, 2006.

AUBERTIN, Catherine et al. (dir.). Les marches de la biodiversité. Paris: IRD Éditions, 2007.

. L'accès aux ressources génétiques et le partage des avantages: une question conflictuelle - exemples du Brésil et de la Bolivie. In: AUBERTIN, Catherine et al. (dir.). Les marches de la biodiversité. Paris: IRD Éditions, 2007, 121-147.

AUBERTIN, Catherine \& MORETTI, Christian. La biopiraterie entre illégalité et illégitimité. »In: AUBERTIN, Catherine et al. (dir.). Les marches de la biodiversité. Paris: IRD Éditions, 2007, 91-120.

BARBOSA, Denis B..Uma introduçāo à propriedade intelectual. Rio de Janeiro: Lumen Juris, 2003.

BAYLĀO, Raul S. \& BENSUSAN, Nurit. A questão das proteção dos conhecimentos tradicionais associados aos recurssos genéticos nos fóruns internacionais. In: BENSUSAN, Nurit \& LIMA, André (dir.). Quem Cala Consente? Subsídios para a proteção aos conhecimentos tradicionais. São Paulo: Instituto Socioambiental, 2003, 17-22.

BOISVERT, Valérie \& CARON, Armelle. Valorisation économique des ressources et nouveaux marchés. In : AUBERTIN, Catherine et al. (dir.). Les marches de la biodiversité. Paris: IRD Éditions, 2007, 195-217.

\& VIVIEN, Franck-Dominique. Un marché pour la biodiversité. In : AUBERTIN, Catherine et al. (dir.). Les marches de la biodiversité. Paris: IRD Éditions, 2007, 223-243.

\section{LA COMMISSION MONDIALE SUR L'ENVIRONNEMENT ET LE} DÉVELOPPEMENT. Notre avenir à tous. Montréal: Éditions du Fleuve, 1988.

CONFÉRENCE DES NATIONS UNIES SUR LE COMMERCE ET LE DÉVELOPPEMENT. Rapport de la réunion d'experts sur les systèmes et l'expérience des pays en matière de protection des connaissances 
traditionnelles, de l'innovation et des pratiques. TD/B/COM.1/33; TD/B/COM.1/EM.13/3. Genebra, 7 dezembro 2000. [Online] Disponível em: $<$ www.unctad.org $>$. Acesso em abr. 2008.

COP 5. Programme of work on the implementation of article 8(j) and related provisions of the convention on biological diversity -IV, Ways and means. Décision V/16. Nairobi, 26 maio 2000. [Online] Disponível em: $<$ http://www.cbd.int/decisions/?m=COP-05\&id=7158\&lg=0>. Acesso em abr. 2008.

CORREA, Carlos Maria. Traditional knowledge and intellectual property: issues and options surrounding the protection of traditional knowledge - A discussion paper. Genebra: Quaker United Nations Office, 2001. [Online] Disponível em: www.iucn.org/themes/pbia/themes/trade/training/TK\%20and\%20Intellectual\%20 Property.pdf. Acesso em nov. 2007.

(dir.). Temas de derecho industrial y de la competencia: propiedad intelectual y políticas de desarrollo. Buenos Aires: Ciudad Argentina, 2005.

DEL NERO, Patricia Aurelia. A propriedade Intelectual da Biotecnologia. In: CARVALHO, Patricia Luciane (dir.). Propriedade intelectual: estudos em homenagem à professora Maristela Basso. Curitiba : Juruá, 2005, 349-361.

MORETTI, Christian \& AUBERTIN, Catherine. Stratégies des firmes pharmaceutiques : la bioprospection en question. In: AUBERTIN, Catherine et al (dir.). Les marches de la biodiversité. Paris: IRD Éditions, 2007, 27-54.

MÜLLER, Andréia N.. A proteçāo dos conhecimentos tradicionais por meio das indicações geográficas. In: RODRIGUES JR, Edson B. \& POLIDO, Fabrício (dir.). Propriedade intelectual: novos paradigmas internacionais, conflitos e desafios. Rio de Janeiro: Elsevier, 2007, 301-346.

ORGANISATION DES NATIONS UNIES. Convention sur la diversité biologique. Rio de Janeiro, 5 junho 1992. [Online] Disponível em: <www.cbd.int $>$. Acesso em: jan. 2008.

Déclaration de Johannesburg sur le développement durable. Johannesburg 4 setembro 2002. UN Doc. A/Conf.199/L.6/Rev2. [Online] Disponível www.rrcap.unep.org/wssd/Political\%20declaration_4\%20Sep\%2002.pdf. Acesso em: jan. 2008.

Déclaration de Rio sur l'environnement et le développement. Rio de Janeiro, 13 junho 1992. UN Doc A/CONF.151/5/Rev. [Online] Disponível em: <www.un.org>. Acesso em: jan. 2008.

ORGANISATION MONDIALE DE LA PROPRIÉTÉ INTELLECTUELLE. [Online] Disponível em: <www.wipo.int>. Acesso em: nov. 2007. 
- Convention de Berne pour la protection des œuvres littéraires et artistiques. Berna, 9 setembro 1886 (ultima modificação : 28 setembro 1979). [Online] Disponível em: <www.wipo.int>. Acesso em: jan. 2008.

. Intergovernmental committee on intellectual property and genetic resources, traditional knowledge and folklore, The Protection of TRADITIONAL KNOW Mechanisms Document prepared by the Secretariat LEDGE: Revised Outline of Policy Options and Legal. WIPO/GRTKF/IC/9/INF/5. Genebra, 27 março $2006 . \quad$ [Online] Disponível em: $<$ http://www.wipo.int/edocs/mdocs/tk/en/wipo_grtkf_ic_9/wipo_grtkf_ic_9_inf_5. doc>. Acesso em: abr. 2008.

Survey on Existing Forms of Intellectual Property Protection for Traditional Knowledge. Document WIPO/GRTKF/IC/2/5. [Online] Disponível em: <www.wipo.int>. Acesso em: abr. 2008.

World Intellectual Property Organization (WIPO) Draft Report on Fact-

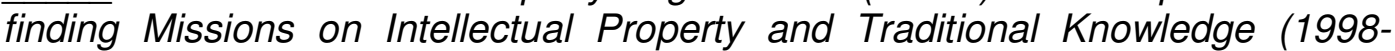
1999) - Draft for Comment - July 3, 2000. [Online] Disponível em: $<w w w . w i p o . i n t>$. Acesso em : nov. 2007.

ORGANISATION MONDIALE DU COMMERCE. Accord instituant l'Organisation mondiale du commerce. Marrakech, 12 abril 1994. [Online] Disponível em: www.wto.org. Acesso em : jan. 2008.

. Accord sur les aspects des droits de propriété intellectuelle qui touchent au commerce. Marrakech, 12 abril 1994. [Online] Disponível em: www.wto.org. Acesso em : jan. 2008.

. Déclaration ministérielle. WT/MIN(01)/DEC/1. Doha, 20 novembro 2001. [Online] Disponível em:. <www.wto.org>. Acesso em : abr. 2008.

PINTON, Florence \& GRENAND, Pierre. Savoirs traditionnels, populations locales et ressources globalisées. In : AUBERTIN, Catherine et al (dir.). Les marches de la biodiversité. Paris : IRD Éditions, 2007, 165-194.

PROGRAMME DES NATIONS UNIES POUR L'ENVIRONNEMENT/CONVENTION SUR LA DIVERSITÉ BIOLOGIQUE Traditional knowledge and biological diversity: Note by the Executive Secretary. UNEP/CBD/TKBD/1/2. Madrid, 18 outubro 1997. [Online] Disponível em: <http://www.cbd.int/doc/meetings/tk/wstkbd-01/official/wstkbd-01-02-en.pdf>. Acesso em: abr. 2008.

SACHS, Jeffrey. O fim da pobreza. São Paulo: Companhia das Letras, 2005.

SANTÉ CANADA. Biotechnologie. [Online] Disponível em: < http://www.hcsc.gc.ca/sr-sr/biotech/index-fra.php>. Acesso em: jun. 2008. 
SHIVA, Vandana. Bioporataria: a pilhagem da natureza e do conhecimento. Petrópolis: Vozes, 2001.

SILVA, Letícia B.. É possível negociar a biodiversidade? Conhecimentos tradicionais, propriedade intelectual e biopirataria. In: BARRAL, Welber \& PIMENTEL, Luiz Otavio (dir.). Propriedade Intelectual e desenvolvimento. Florianópolis: Fundação Boiteux, 2006, 299-328.

VARELLA, Marcelo D.. Direito internacional econômico ambiental. Belo Horizonte: Del Rey, 2004.

ZAZZALI, Jorge C.. Propiedad intelectual, diversidad biológica y conocimentos tradicionales - una visión desde los Andes y la Amazonia. In: CORREA, Carlos Maria (dir.). Temas de derecho industrial y de la competencia: biotecnología y derecho. Buenos Aires: Ciudad Argentina, 1997, 89-130.

Revista Brasileira de Direito Internacional, Curitiba, v.7, n.7, jan./jun.2008 